\title{
Civilisations
}

Revue internationale d'anthropologie et de sciences

humaines

56 | 2007

Après la catastrophe

\section{«Ici nous troquons ton désespoir pour de l'espérance ... » : approche ethnographique du troc urbain dans une société en crise}

\section{Olivia Angé}

\section{(2) OpenEdition}

\section{Journals}

\section{Édition électronique}

URL : http://journals.openedition.org/civilisations/1517

DOI : 10.4000/civilisations. 1517

ISSN : 2032-0442

\section{Éditeur}

Institut de sociologie de l'Université Libre de Bruxelles

\section{Édition imprimée}

Date de publication : 1 décembre 2007

Pagination : 63-85

ISBN : 2-87263-016-3

ISSN : 0009-8140

\section{Référence électronique}

Olivia Angé, « «Ici nous troquons ton désespoir pour de l'espérance ... » : approche ethnographique du troc urbain dans une société en crise », Civilisations [En ligne], 56 | 2007, mis en ligne le 01 décembre 2010, consulté le 30 avril 2019. URL : http://journals.openedition.org/civilisations/1517 ; DOI : 10.4000/civilisations. 1517 


\title{
"Ici nous troquons ton désespoir pour de l'espérance ... " Approche ethnographique du troc urbain dans une société en crise
}

\author{
Olivia ANGÉ
}

Résumé : Cet article est consacré à la présentation d'un réseau de troc urbain qui s'est déployé à l'initiative de la classe moyenne argentine, suite à la crise générale qui a traversé le pays en 2001. Une première partie est consacrée à la description du contexte social dans lequel le réseau a pris son essor. Nous rendons ensuite compte de son évolution à partir du projet utopique des pères fondateurs. Enfin, deux descriptions ethnographiques permettent au lecteur d'imaginer en quoi consiste concrètement ce système économique informel à travers lequel ont été introduits des bons d'échange reconnus à l'échelle nationale.

Mots-clés : Argentine, troc, société civile, crise économique, échanges.

Summary: This article is devoted to a description of the network of urban exchange which has spread through the initiative of the Argentinian middle-class, following a general crisis that crossed the country in 2001. The first part is dedicated to the description of a social context in which the network has expanded rapidly. I then account for its evolution from the utopic project of its founding fathers. Finally two ethnographical descriptions allow the reader to imagine what concretely makes up this informal economic system through which exchange bonds have been introduced and are now recognised at a national level.

Key words: Argentina, barter, civil society, economic crisis, exchanges. 
T orsque je suis allée dans ce pays en février 2003, ce n'était plus le cas. Emportée

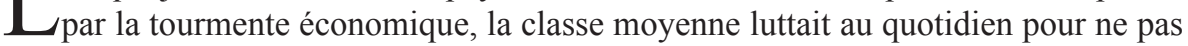
devenir aussi pauvre que les plus pauvres qui, hier encore, représentaient à ses yeux la honte de la nation. Le marché informel constituait désormais un secteur prometteur pour qui entendait mettre sa débrouillardise à profit, à tel point que l'on put observer des initiatives citadines visant à «formaliser l'informel». C'est ce dont j'ai été témoin lorsque je me suis intéressée au troc urbain. J'ai centré mon étude sur la province de Buenos Aires, dans laquelle j'ai réalisé la majeure partie de mes enquêtes ${ }^{1}$. Pendant deux mois, je me suis entretenue avec des acteurs de statuts divers et j'ai réalisé des observations, participantes ou non, dans différents clubs regroupés dans le Réseau Global de Troc (Red Global de Trueque). Dans le club Color Esperanza, pendant un mois et demi, j'ai préparé des cakes, que j'échangeais contre des produits généralement alimentaires. Parallèlement à ces activités, je me suis intégrée au groupe des organisateurs -j'entends par « organisateur », tout membre du réseau qui joue un rôle dans la mise en place ou dans le maintien de sa structure. J'ai ainsi côtoyé les fondateurs du réseau, que j'observais par ailleurs à l'action dans le club dont ils assuraient la coordination. Le caractère récurrent de mes prestations les a rendues ordinaires. Une fois que ma présence n'attira plus les regards, j'ai pu participer de manière naturelle aux multiples conversations qui rythmaient les foires de troc. Après quelques réunions, je connaissais l'histoire des membres réguliers; bientôt j'allais pouvoir retracer des parcours de vie.

Dans cet article, je rendrai compte des résultats de ce travail ethnographique en m'interrogeant sur la nature des échanges mis en place. Les pratiques économiques observées ont varié en fonction des relations qui se sont instaurées entre les protagonistes, celles-ci dépendant elles-mêmes de la prégnance du cadre idéologique du trueque ${ }^{2}$ au sein de la sphère d'échange. Nous pourrons dès lors nous demander s'il faut voir ce système comme composé d'échanges marchands médiatisés par les "creditos », cette monnaie spécifique imaginée pour répondre à la crise ou plutôt de prestations sociales totales, au sens défini par Marcel Mauss (2004 : 147). Mais avant d'aborder ces questions, il nous faut souligner ici comment l'analyse des échanges de trueque nous a servi de révélateur de la capacité humaine d'innovation et d'adaptation après ce qui a pu apparaître comme l'ébranlement des fondements idéologiques et économiques de la société argentine.

\section{Contexte historique}

La « catastrophe argentine » est partie d'une crise économique pour s'étendre aux sphères politique et sociale. La dégradation de la situation économique, dont les signes étaient devenus évidents à la fin des années quatre-vingt-dix, annonçait les événements à venir. Il a pourtant fallu attendre encore plusieurs années pour que la société civile se manifeste face au système politique mis en place par le président Carlos Menem.

1 Cette précision a son importance, car ni la crise ni le troc ne se présentent de manière uniforme dans l'ensemble de cet immense pays.

2 Dans le texte, nous avons gardé le terme espagnol de trueque afin de distinguer les échanges profondément hétérogènes qui se sont déployés au sein du réseau de ce que recouvre habituellement le terme de " troc », lequel renvoie à un échange de biens ne faisant appel à aucun instrument monétaire. 
Ce dernier avait gagné le pouvoir en 1991 en séduisant par son discours péroniste une population appauvrie par l'hyperinflation survenue en $1989^{3}$. Il affirmait détenir les solutions qui permettraient de redresser la situation et, dans une certaine mesure, ce fut le cas, bien que sa politique n'ait pas toujours été en adéquation avec les objectifs péronistes annoncés. Parmi ses remèdes à l'inflation, il préconisait l'arrimage du peso au dollar à travers le Plan de convertibilité. A long terme, cette mesure devait conduire à une atrophie de la production nationale au profit du commerce extérieur. Il était désormais plus rentable de faire venir les marchandises des pays voisins que de mettre à profit la force de travail nationale, et les petites et moyennes entreprises ne purent longtemps résister à la concurrence des produits importés. La sortie de la crise de 1989 mettait donc en place les éléments structurels de la catastrophe à venir. Mais au début des années quatre-vingt-dix, la population avait confiance en son président qui avait alors toutes les allures d'un sauveur. Semblant convaincue qu'il était effectivement capable de transformer les pesos en dollars, elle refusait de renoncer à la réalisation d'un rêve inscrit dans son imaginaire : l'entrée imminente dans le «premier monde » prédite par le président. Pour cette population d'immigrés d'origine européenne, le projet était empreint d'une charge affective particulière : il redonnait l'espoir d'un retour à la culture originelle. Les Argentins voulaient devenir «modernes » et tout se passait comme si leurs désirs devenaient réalité. L'invasion du marché par les produits importés offrait enfin l'accès à une consommation à l'occidentale. Les yeux rivés sur le Nord, la société suivait de près les dernières nouveautés du " premier monde » qui représentait la référence du goût légitime. "Le changement fut impressionnant. Avant cela nous étions comme la Bolivie » (Geronimo, 24 ans, étudiant en droit).

Après une période pleine d'assurance pendant laquelle les promesses présidentielles semblèrent se réaliser, le coût de cette politique commença à se faire sentir : augmentation de la dette publique, dépendance vis-à-vis des entreprises internationales, expansion du chômage, désarticulation de l'Etat, concentration des richesses. En 1998, après avoir privatisé l'ensemble du patrimoine public, Menem remit à Fernando de la Rua un pays en très mauvaise posture. Trois ans plus tard, les plus intuitifs (ou les mieux informés), vidaient brutalement leurs comptes bancaires. Les épargnants de la classe moyenne et les moins nantis furent, quant à eux, piégés par le gel des dépôts bancaires ou " corralito »" Quelques mois plus tard, le Président de La Rua s'enfuit soudainement de la Maison Rose en hélicoptère. Pendant deux semaines, les chefs d'Etat se succédèrent, décrédibilisant encore plus l'élite politique. L'espace public fut investi par les ménagères qui clamaient leur colère au rythme des cacerolazos : " ; Que se vayan todos!" (Qu'ils s'en aillent tous !) criaient-elles aux politiciens en brandissant bruyamment leurs casseroles vides, symboles de la pénurie. La crise, jusqu'alors essentiellement économique, gagna les sphères politique et sociale et le processus de désinstitutionalisation s'emballa.

En janvier 2002 arriva ce que personne n'avait osé imaginer : Eduardo Duhalde, qui était alors au pouvoir, prit la décision de libérer le peso de son arrimage au dollar. La population perdit confiance en la monnaie nationale, symbole de la puissance de l'Etat

3 A cette époque, l'économie nationale payait toujours les frais de la politique économique mise en œuvre par le gouvernement militaire expulsé du pouvoir en 1983, après sept années de dictature sanglante. 
et de sa souveraineté. La dévaluation consécutive à la mise en place du corralito fut interprétée comme "une gigantesque attrape » (Diego, 36 ans, indépendant) orchestrée par les politiciens. Du jour au lendemain, le fruit de longues années d'épargne avait perdu près de la moitié de sa valeur. Ces différentes mesures unirent la classe moyenne dans une nouvelle communauté de destin. «Même si une grande majorité ressent de la haine et de la honte à ne plus pouvoir faire ce qu'elle faisait avant, elle continue à s'identifier comme classe moyenne et éprouve une véritable terreur de la misère » (Clarín, 03/09/2002). La perte de pouvoir d'achat entre 1998 et 2003 fut estimée à 50\%. En octobre 2002, 57,5\% de la population vivait en dessous du seuil de pauvreté et $27 \%$ de ces « pauvres » étaient considérés comme indigents (Gabetta, 2003 : 14). Les déclassés se mobilisèrent face à la crise et occupèrent la scène publique. Les exemples de leur activisme sont innombrables. Citons le cas des municipalités envahies par les citoyens révoltés barrant l'accès aux fonctionnaires, des usines en faillite réinvesties par des travailleurs clandestins, ou encore des assemblées de quartier qui se constituaient à chaque coin de rue. Les réseaux de troc font partie de ces initiatives.

La dérégulation du marché de l'emploi joua un rôle de catalyseur dans les mises en crise individuelles. Obligé de se rabattre sur des produits moins valorisés, le « citoyenconsommateur » qui avait été mis en exergue sous la présidence de Menem dut s'adapter à la pénurie en changeant son style de vie, ce qui affecta son identité sociale. En perturbant la division sexuelle et générationnelle du travail, ces problèmes économiques eurent des conséquences importantes sur la structure familiale : "On m'a appris que c'est moi qui dois ramener l'argent à la maison. Imagine-toi la honte que c'est d'envoyer ma fille s'alimenter à la cantine publique. Je n'ai plus de dignité et je suis en train de m'éloigner de mes proches » (Roberto, 50 ans, peintre sans emploi). L'augmentation fulgurante du taux de divorce témoigne de l'apparition de ces nouvelles tensions au sein du foyer. La cohésion de la famille élargie eut également tendance à s'affaiblir. Devenues trop chères, les fêtes qui rythmaient le calendrier ne purent plus toujours être célébrées. Les nourritures festives qui font la fierté d'une famille en lui permettant d'afficher les symboles d'une réussite économique disparurent du quotidien. La solidarité familiale resta néanmoins très forte dans ces moments difficiles. Comme beaucoup de sociétés d'immigrés, les Argentins soulignent l'importance des valeurs familiales dans la représentation qu'ils se font de leur identité, même s'ils n'ont plus les moyens matériels de respecter les comportements normatifs correspondant aux valeurs affichées.

La particularité de la crise argentine, c'est donc l'écroulement d'une puissante classe moyenne. "Avant, il y avait des valeurs comme l'amour ou l'amitié, maintenant, l'unique valeur c'est l'argent » (Aldana, 52 ans, secrétaire sans emploi). C'est aussi la crainte de devoir remettre en question ce qui lui apparaissait comme des évidences :
Notre société était caractérisée par une trame sociale originale, différente de celle des autres pays : notre unité familiale est différente, nos valeurs sont différentes et c'est justement cela que nous sommes en train de perdre. Le respect des personnes âgées, des enseignants, des policiers... tout cela faisait partie de notre patrimoine. Nous étions tellement fiers de célébrer les anniversaires en famille, les mariages, les repas du dimanche... d'être un peuple affectueux. C'est notre valeur ajoutée qui disparaît parce que nous sommes en train de perdre l'essentiel : le respect et cette capacité à communiquer notre affection (Andrea, 36 ans, vétérinaire).

La mise à mal du système de valeurs laissa la porte ouverte aux comportements anomiques. La violence urbaine se généralisa dans les rues de Buenos Aires, une ville 
autrefois dépeinte par ses habitants comme « la capitale la plus sûre d'Amérique latine ». La peur qui en résulta eut pour effet de modifier les comportements. Les enfants cessèrent de jouer dans les rues, les voisins mirent fin à leurs interminables discussions de trottoir accompagnées du traditionnel maté. C'est jusque dans l'intimité de leur foyer que les Argentins ressentaient la menace : "Avant je dormais la fenêtre ouverte. Comme ça, tranquillement. Maintenant, je n'ai plus assez d'argent pour agrandir les barrières de la maison et je vis dans une peur perpétuelle " (Paola, 52 ans, troqueuse sans emploi). Le climat de crainte généralisée et la manipulation à laquelle se livrèrent les médias conduisit de nombreux particuliers à se munir d'une arme à feu, ce qui engendra une escalade de la violence. Comme le remarquent Laura Golbert et Gabriel Kessler (2002 : 85-100), le sentiment d'insécurité fut amplifié par le caractère généralisé de la crise : chaque concitoyen devenant un danger physique ou un concurrent économique potentiel, la pénurie éveilla des instincts de survie. Dans un tel climat, tout était permis pour s'en sortir, même enfreindre des lois que les Argentins pensaient inscrites dans leur contrat social. « Si les politiciens nous ont tout volé, pourquoi ne se volerait-on pas entre nous?» se demande Christina (46 ans, troqueuse sans emploi).

Menem occupa le centre d'un fantasme partagé par les déclassés. Persuadés de s'être fait abuser, ils étendirent leur rejet à toute l'élite au pouvoir. L'appareil politique leur paraissait profondément corrompu et ils formulèrent des accusations qui, véhiculées sous la forme de rumeurs, emportaient l'adhésion générale : "A Buenos Aires, tout le monde sait que Duhalde est à la tête du trafic de drogue » (Diego). Cette culture de la défiance est ancrée dans l'histoire du pays. Les souvenirs de la dictature attisaient la suspicion à l'égard de politiciens souvent considérés comme les bourreaux de la nation. Mais, si de nombreux méfaits étaient imputés aux politiciens, les citoyens n'échappaient pas à un certain sentiment de culpabilité. La société se sentait victime d'elle-même, car « elle continuait à voter pour les mêmes escrocs » (Guillermo Magadan, 40 ans, technicien de télévision). Par ailleurs, certains interprétaient la crise et son caractère répétitif dans l'histoire nationale comme l'effet d'une idiosyncrasie argentine: "Des politiciens corrompus, il y en a partout dans le monde. Le vrai problème c'est que l'Argentin est arnaqueur, voleur et menteur » (Jeronimo). La population ne semblait cependant pas prête à accepter ces cycles comme une fatalité. Même si la catastrophe était une constante dans l'histoire nationale, elle provoquait toujours le même effet de surprise à l'échelle des histoires individuelles : Comment imaginer en effet un retour en arrière après avoir goûté cette vie « modernisée » tant convoitée?

\section{Quand la catastrophe ouvre les portes de l'utopie}

C'est sur ce terrain dévasté que s'est développé le Réseau Global de Troc, dont il sera maintenant question. Il résulte de l'initiative de trois intellectuels vivant à Bernal, dans la banlieue de Buenos Aires : Ruben Ravera, Carlos De Sanzo et Horacio Covas. Carlos De Sanzo est psychologue, mais il n'exerce plus sa profession, car il a progressivement perdu sa clientèle en raison de l'instabilité économique. Avec Ruben Ravera, le directeur du Musée Hudson, il avait aménagé un potager "comme celui de la petite ferme de style européen de son enfance ». Les deux amis ont donné ensemble divers cours d' « autosuffisance familiale ». C'est comme cela qu'ils ont rencontré Horacio Covas, un chimiste récemment licencié de son poste de cadre dans une entreprise de cosmétiques. 
L'histoire remonte à 1989, époque où l'hyperinflation avait plongé de nombreux foyers dans une pauvreté extrême. Dans la province de Buenos Aires s'étaient mis en place des programmes visant à promouvoir la production domestique et communautaire. A cette époque, Ruben Ravera et Carlos De Sanzo avaient fondé ensemble le Programme d'Autosuffisance Régionale, qui leur avait permis d'instaurer différents projets, tels que le recyclage de l'eau de pluie et l'utilisation de l'énergie solaire et éolienne. La réalisation d'un « technopotager» (tecnohuerta) visait à démontrer qu'il était possible de produire des aliments sur une surface réduite en combinant la technologie moderne et des principes d'agriculture biologique. C'est ainsi que Carlos, qui cultivait des citrouilles sur sa terrasse, obtint une récolte à ce point prolifique qu'il put venir en aide à une voisine. C'étaient les prémisses d'un troisième projet, associant Horacio Covas. Il visait à mettre en place un système économique permettant d'échanger les surplus de la production domestique. L'ensemble du programme avait pour ambition de remettre la production domestique à l'ordre du jour et de créer des surplus destinés à l'échange.

Si l'initiative résulte d'un concours de circonstances, il n'en demeure pas moins que les trois collaborateurs lestèrent leur projet d'un bagage théorique ambitieux. Ces ambitions, ils m'en ont fait part lors d'un entretien réalisé à Buenos Aires en avril 2003 :

\begin{abstract}
Notre modèle économique est révolutionnaire. Il propose d'intégrer au marché l'humanité entière en offrant la possibilité d'être productif à partir du seul capital humain. Le troc est une économie de l'interstice, il permet de socialiser ceux qui ont été laissés en dehors du processus de mondialisation.
\end{abstract}

En ouvrant le premier club en 1995, ils entendaient " profiter des excédents des uns et les transformer en offre pour d'autres sans qu'intervienne l'argent dans la capacité d'accéder aux biens et aux échanges » (entretien d'avril 2003). C'est à cet effet qu'ils réunirent dans leur garage une vingtaine de voisins préoccupés comme eux par la dégradation de leur environnement naturel et économique. Les premiers échanges, essentiellement alimentaires, se faisaient de manière directe; ils étaient néanmoins comptabilisés dans un carnet (et plus tard dans un ordinateur), afin que le troqueur puisse consommer les produits de son choix sans avoir à assumer les obligations d'une réciprocité immédiate. Ce troc était défini comme «multiréciproque », l'équilibre étant réalisé à l'échelle du groupe et non par le truchement de transactions bilatérales. A la fin, la valeur totale de la production et de la consommation de chacun était comptabilisée afin d'assurer une répartition équilibrée des quantités produites. Petit à petit, le nombre de participants augmenta et l'offre se diversifia : après les articles alimentaires apparut l'artisanat, puis les services. La dynamique était lancée, la diversification attirant des nouveaux venus, qui à leur tour amplifièrent la diversité. Lorsque le nombre de participants devint trop important, il fallut trouver un autre moyen de répertorier les échanges. A la recherche d'un système décentralisé, les fondateurs (qui se dénommèrent Conseil Assesseur) instaurèrent un système de bons mentionnant les noms du créditeur et du débiteur. Non transférables à l'origine, ces « petits papiers » le devinrent rapidement et furent utilisés comme monnaie communautaire valable à l'intérieur du club, un groupe encore restreint caractérisé par la confiance mutuelle régnant entre ses membres. C'est ainsi que naquit une nouvelle monnaie à usage local, le credito, dont l'usage se généralisa progressivement à l'échelle régionale, puis nationale.

Les clubs se multiplièrent par scission et par imitation. Le réseau qui naquit de leurs interactions fut baptisé Réseau Global de Troc. Les clubs devinrent des nodos (nœuds) au 
sein desquels se déroulaient les interactions entre les membres du réseau. Entre 1995 et 1997, la croissance du réseau resta modérée et restreinte à la capitale. Le nombre de nodos était estimé à deux cents, pour dix mille participants. La masse monétaire en circulation devait approcher les deux cent mille unités, puisque chaque nouveau venu se voyait attribuer vingt creditos ${ }^{5}$ pour mettre en place son système productif. L'impression des creditos relevait de la compétence des fondateurs, qui l'exécutaient de manière artisanale. Quelques presses à billets installées dans un hangar suffirent à la création d'une monnaie diffusée à l'échelle nationale.

A la fin de la seconde année, le soutien des médias vint renforcer le mouvement d'adhésion et provoqua une première expansion brutale du troc tant dans la capitale que dans les provinces. De nouveaux réseaux se constituèrent partout, tandis qu'apparaissaient des nodos indépendants. A la période de centralisation succéda une période de régionalisation pendant laquelle des «commissions de creditos » furent constituées afin de réguler l'émission des bons, tandis que des assemblées régionales se chargeaient de diverses tâches matérielles et assuraient la diffusion des principes éthiques. Des réunions mensuelles furent organisées dans les nodos, puis dans les différentes zones et enfin, entre les zones.

Après s'être consolidé de l'intérieur, le réseau commença à établir des relations avec l'Etat et le marché formel. A ce stade, il incorpora des petites et moyennes entreprises du secteur formel. Les activités et les statuts des entreprises affiliées étaient variables : certaines se bornaient à développer le troc, d'autres jouissaient d'une flexibilité leur permettant d'entrer et de sortir de la sphère formelle selon la conjoncture. D'autres encore s'enracinaient dans le troc avant d'affronter l'impitoyable concurrence du marché. Certaines, proches de la faillite, se donnaient l'occasion de revitaliser leurs activités en bénéficiant de cet immense réseau d'échange. Celles qui étaient déjà en faillite pouvaient être réinvesties par la force de travail des troqueurs, qui leur offraient ainsi une reconversion dans l'informel. Mentionnons encore le cas des entreprises qui développaient des partenariats avec un ou plusieurs clubs, ou avec le réseau dans son ensemble (la carte d'affiliation au club permettait à son détenteur de bénéficier de réductions dans certains commerces du secteur formel). Il était aussi possible de payer une partie de son dû en creditos. Ces collaborations étaient précieuses, car elles donnaient accès à des produits que l'on trouvait difficilement dans l'économie informelle (presse, médicaments, cartes téléphoniques...).

En 1997, un débat s'engagea avec l'Etat en vue d'obtenir la reconnaissance formelle de cette nouvelle sphère économique en pleine expansion. Le gouvernement de la ville de Buenos Aires fut le premier à appuyer ouvertement cette initiative. Il fut suivi par d'autres villes et provinces, qui la déclarèrent d'intérêt collectif. En 2000, le ministère de l'Economie de la Nation reconnut le troc comme une économie alternative apte à pallier les dysfonctionnements du marché de l'emploi - le soutien qu'il apportait à l'économie familiale était déjà substantiel : il dépassait très largement le salaire minimum. Cependant, ces discours n'aboutirent à aucune reconnaissance formelle, si bien que le trueque resta confiné dans un espace qui, au grand regret des fondateurs, n’était pas balisé par la loi.

5 Les chiffres avancés dans ce paragraphe viennent de l'article de Laurent Montillet (2004). Ils sont à interpréter avec précaution; il s'agit d'estimations en l'absence d'un recensement rigoureux, et l'auteur nous met lui-même en garde quant à leur degré de précision : les nœuds naissent et meurent sans toujours en aviser l'organisation, et certains sont totalement indépendants. 
En 1999, le Conseil Assesseur imposa l'établissement d'un contrat avant l'ouverture de tout nouveau club, et ceci afin d'homogénéiser les pratiques à l'échelle nationale. A partir de ce moment, les coordinateurs durent se voir reconnaître une qualification pour pouvoir revendiquer leur appartenance au Réseau Global de Troc. Cette franchise portait également sur l'émission des creditos, que les fondateurs entendaient uniformiser, centraliser et financer. Chaque membre se voyait dorénavant attribuer un capital de cinquante creditos vendus au prix de deux pesos - il pouvait se les procurer à la « banque centrale » où ils étaient imprimés, ou auprès du coordinateur de son club ${ }^{6}$. C'est aussi à cette époque que la masse des creditos mis en circulation commença à enfler et que l'unité perdit de sa valeur. Pour leurs détracteurs, les fondateurs en auraient émis une quantité excessive pour réaliser un profit sur la transaction.

Entre janvier 2001 et avril 2002, à la suite de la crise de décembre 2001, le nombre de participants augmenta de manière exponentielle. Lorsque l'Etat se révéla incapable de distribuer les aides aux foyers sans revenus, les classes les plus défavorisées virent leur situation économique, déjà précaire, se détériorer et l'on assista à l'émergence d'une situation nouvelle. La consommation des biens de trueque avait représenté jusqu'alors une alternative écologique et éthique pour une classe moyenne appauvrie en quête de distinction ${ }^{7}$. Avec l'afflux des plus démunis, les clubs et les foires renvoyaient désormais l'image d'une sphère économique de seconde zone. Sachant, comme Pierre Bourdieu l'a montré (1979), que la consommation est un marqueur symbolique du groupe social d'appartenance, un tel amalgame était difficilement acceptable par la classe moyenne. C'est l'époque où le credito devint la monnaie du pauvre. L'effet de distinction culturelle s'en trouva anéanti et les premiers adhérents furent les premiers à déserter quelques mois plus tard. Mais avant cela, le réseau s'était étendu de manière brutale et avait dépassé largement les frontières argentines. Des clubs étaient nés dans les pays limitrophes (Chili, Uruguay, Brésil, Bolivie), mais également en Equateur, en Colombie ou en Espagne. En Argentine, leur nombre était alors estimé à sept mille pour un total de six millions de participants. La masse de bons en circulation était évaluée à deux cents millions. En novembre 2002, le quotidien Clarín annonçait qu'il était possible de se procurer en creditos une voiture, un terrain, une séance de psychanalyse, une consultation chez le dentiste, un voyage en bus pour l'Uruguay ou des places de théâtre.

Sous sa forme idéale, le troc était une réponse à la crise nationale. Cependant, les dérives n'avaient pas tardé à apparaître, faisant de ce secteur le creuset de pratiques opportunistes bien éloignées de ce qu'avaient imaginé les pionniers. Il en résulta une perte de confiance générale de la part des participants, qui désertèrent leurs clubs de troc. Les dysfonctionnements résultaient d'une part de problèmes d'organisation interne et, d'autre part, des relations qu'entretenait le réseau avec le secteur formel.

En 2002, apparurent les premières falsifications. L'invasion des nodos par cette « fausse monnaie » provoqua une inflation, elle-même amplifiée par l'inflation que connaissait le secteur formel à la suite de la dévaluation du peso. Le mouvement s'emballa : un total de cinq cents millions de creditos falsifiés aurait été injecté dans le système. Selon le quotidien Clarín, dans certaines régions, les billets falsifiés représentaient jusqu'à $90 \%$ des bons en

6 Ces nouvelles mesures concernant la distribution des creditos entraînèrent des désaccords provoquant différentes scissions au sein du réseau.

7 Voir à ce propos Inés Gonzalez Bombal (2002). 
circulation. La collaboration avec le marché formel par le truchement des entreprises intégrées au réseau fut la première victime de la décrédibilisation du credito. Différentes mesures de protection furent prises par les fondateurs pour mettre fin aux falsifications, mais le manque de communication au sein du réseau en compromit l'efficacité.

A la croissance exponentielle du réseau succéda une décroissance aussi soudaine : le nombre de participants tomba à un million pour deux à trois mille clubs encore actifs. Les rumeurs se mirent à circuler; chacun avait sa propre version des faits. Certains y voyaient la conséquence de la falsification du credito, d'autres un complot de l'Etat ou du FMI, d'autres encore, un problème de contrôle des produits en circulation ou l'effet d'une corruption généralisée. Au-delà des divergences d'interprétation, la crise était effective ; elle se marquait dans le langage des troqueurs. Les expressions qu'ils utilisaient pour décrire les crises des marchés formel et informel étaient identiques : l'augmentation des prix, la rareté, la généralisation de l'agressivité et la diffusion d'un individualisme exacerbé étaient soulignés.

\section{La société idéale selon les trois fondateurs : les ambitions originelles}

Si l'idée d'échanger le surplus de la production domestique est le fruit d'un concours de circonstance, le déploiement du Réseau Global de Troc ne résulte pas d'une évolution fortuite. Carlos, Ruben et Horacio revendiquent une planification consciente et énumèrent fièrement leurs auteurs de prédilection : Marx, Keynes, Toffler ${ }^{8}$. Ce n'est sans doute pas un hasard s'ils me proposèrent comme premier lieu de rencontre la fameuse brasserie Tortoni, quartier général des intellectuels de l'Argentine prospère, pour m'exposer le modèle de société qu'ils avaient imaginé pour un monde meilleur. Au fil de l'entretien, j'ai compris que ce que les fondateurs avaient en vue n'était pas seulement une nouvelle forme d'organisation des échanges économiques, mais un projet de société qui pallierait les dérives d'un capitalisme jugé responsable des maux de leur nation. S'ils qualifièrent leur réseau de " global », c'est d'ailleurs en allusion ironique au processus qu'ils entendaient combattre. Ce n'est d'ailleurs pas par hasard qu'ils qualifièrent leur réseau de « global », mais bien en allusion ironique au processus qu'ils entendaient combattre. Ils rêvaient d'une société «à dimension humaine " régie par des considérations d'ordre social et écologique, par opposition au « monde capitaliste » dans lequel la sphère économique avait pris trop d'ampleur. Ils pensaient qu'un retour au communautaire permettrait une prise d'autonomie par rapport à l'appareil étatique, considéré comme le principal responsable des malheurs argentins. Pour les fondateurs, le slogan ; Que se vayan todos ! ne renvoyait pas à une utopie, mais plutôt à une possible sortie de la catastrophe nationale.

C'est de cette vision de la société idéale dont il sera question dans les paragraphes suivants. Notons que les membres fondateurs remanient constamment le cadre normatif en fonction des défis auxquels ils sont confrontés. Les lignes qui suivent exposent leur position en avril 2003, à l'heure de la reconstruction.

8 Le porte-parole du réseau cite une série de grands noms de multiples disciplines (de la sociologie à l'économie en passant par la biologie et la psychologie), dont les théories auraient pu constituer un fondement épistémologique au système d'échange mis en pratique. Néanmoins, les fondateurs aiment à souligner qu'ils ne s'inscrivent dans aucun courant spécifique. Revendiquant le caractère inédit du système dont ils seraient les seuls créateurs, ils affirment avoir longtemps ignoré l'existence d'expérience similaire sur les autres continents. 
Certains aspects du réseau sont inspirés de la structure des groupes d'entraide du type Alcooliques Anonymes, ce qui témoigne de l'influence de la pratique de psychologue de Carlos. Ce dernier est à l'origine d'une nouvelle thérapie destinée à guérir ce qu'il appelle l'«addiction pécuniaire»: les Narcotiques Anonymes. Je fais part ici de ses convictions :
L'Union Européenne et le Fonds Monétaire International nous ont drogués à l'argent et nous sommes maintenant des Narcotiques Anonymes. Nous voulons aider les gens à en finir avec cette addiction pécuniaire. L'argent est comme une drogue en raison des intérêts qu'il génère. Par exemple, lorsque j'utilise ma carte de crédit, je dois payer un intérêt de $6 \%$ sur ma consommation en pesos et $10 \%$ sur ma consommation en dollars. Cela signifie que chaque mois, je dois sortir dans la rue chercher de l'argent ou le ravir à quelqu'un d'autre afin de pouvoir le payer, comme un drogué qui part voler pour payer sa drogue. Ce que nous proposons, c'est une monnaie qui ne soit pas addictive et qui renverse la dynamique usuraire de l'argent.

Les douze principes qui constituent le " code du treuque ${ }^{9}$ dont l'acceptation est censée conditionner l'intégration au réseau sont un reflet de ce modèle thérapeutique. Dans le premier principe, les fondateurs réaffirment leur défiance à l'égard de l'argent : "Notre pleine réalisation d'être humain ne doit pas être conditionnée par l'argent ». Les conditions d'adhésion sont peu restrictives, il faut: " assister aux réunions, participer au cours d'initiation, être producteur et consommateur de biens, de services ou de savoir dans le respect des règles de qualité, et s'aider soi-même " (cinquième principe). Pour le reste, le réseau est ouvert à tous. Les derniers principes soulignent son caractère démocratique et l'autonomie qu'il implique et engendre.

Le concept de « prosommateur», emprunté à Alvin Toffler (1980), est essentiel. Ce néologisme désigne tout troqueur qui produit et consomme en quantités équivalentes dans l'espace de la feria (foire). En « prosommant» dans la même entité sociale, les partenaires évitent les déséquilibres économiques, puisque la sphère dans laquelle les producteurs peuvent écouler leurs denrées est celle-là même qui permet aux consommateurs de subvenir à leurs besoins. Nous comprenons désormais pourquoi les fondateurs faisaient de la structuration de la société en nodos la solution à la pénurie. En pratique, pour être un bon prosommateur il faut consommer une quantité égale à la production que l'on a écoulée. Ceci afin d'éviter une accumulation des creditos qui nuirait au système en amenuisant la circulation des biens, d'une part, de donner aux troqueurs une maîtrise sur leur niveau de vie, d'autre part : pour avoir accès à une plus grande quantité de biens, il leur suffit de produire plus. Pour les organisateurs, il s'agit de raviver la culture du travail que beaucoup de citoyens auraient perdue malgré eux, et ils présentent une vision ludique du travail, consistant avant tout à se perfectionner dans une activité que l'on apprécie (cuisine, couture, agriculture...). La notion d'emploi et les relations de subordination qui en découlent disparaissent dans ce nouveau cadre de production. En incitant chacun à retrouver ses capacités à produire dans l'espace domestique et à échanger le surplus avec les membres de son nodo, les organisateurs entendent mettre en valeur un « capital humain » occulté par le mode de production capitaliste. Il est par ailleurs spécifié que ces 
échanges, loin d'engendrer un profit matériel, doivent uniquement mettre à la disposition de chacun une plus ample palette de produits de consommation.

S'il est un bon prosommateur, le membre d'un club s'engage à produire et à consommer au sein d'une même entité, et donc aussi à reproduire les échanges avec ses partenaires. La réciprocité est considérée comme une valeur fondamentale. En assurant la répétition des transactions à travers le temps, elle renforcera la confiance et la solidarité entre les membres. Il s'agira de promouvoir un système d'entraide générale au sein duquel les biens, les savoirs et les pouvoirs circuleront sans distinction entre les partenaires, sans que le profit individuel ne constitue la motivation première des échanges.

La responsabilité et l'autogestion constituent des valeurs fondamentales à tous les niveaux du réseau. Chacun des clubs et, à l'intérieur de ceux-ci, chacun des prosommateurs sont ainsi considérés comme des entités autonomes responsables de leur organisation. Cette absence de centralisation devrait permettre aux nodos de s'adapter aux aspirations de leurs membres et de promouvoir l'expression de la démocratie participative. Au sein des clubs, la fonction de coordinateur est tournante et ne donne lieu à aucun privilège d'ordre politique ou économique. Tous les membres, égaux entre eux, doivent pouvoir prendre part à l'organisation et contribuer aux prises de décision. Cette organisation essentiellement horizontale vise également à stimuler la diffusion du modèle en autorisant la reproduction des clubs en l'absence de « représentants officiels ». Elle favoriserait ainsi l'élargissement d'un projet local à l'échelle nationale, voire internationale.

Le credito constitue un élément capital pour la mise en pratique de la thérapie visant à anéantir l'addiction monétaire. S'il est couramment appelé « monnaie », ses créateurs insistent sur le fait qu'il possède des qualités spécifiques permettant de le distinguer du peso national. Cette monnaie thérapeutique est qualifiée de « sociale » pour rappeler ses vertus intégratives, par opposition à l'exclusion sociale dont la devise nationale serait responsable. Ainsi, les fondateurs insistent sans relâche sur le fait que « le credito ce n'est pas de l'argent, c'est un concept totalement différent. Nous, nous l'envisageons comme un outil d'échange » (Horacio Covas, en février 2003). Ces propos sont développés par Suzana Rivano ${ }^{10}$ dans ses cours d'introduction au troc multiréciproque :

Le credito remplace le produit que vous faites, mais il ne répond pas à vos besoins. Avec le credito que vous avez reçu, vous n'allez pas pouvoir manger. Vous troquez réellement quand vous avez rendu vos creditos au réseau et que vous avez ainsi pourvu à vos besoins. Vous êtes obligés de les rendre au réseau parce que sinon vous volez un outil à votre club. Il faut rendre les creditos, c'est une promesse. En les rendant, vous fermez un troc qui était resté ouvert. Ici on ne paie pas!

[photo 1]

A la différence de l'échange par troc direct qui, d'un point de vue strictement économique, laisse les partenaires quittes, il est attendu du troc multiréciproque médiatisé par les creditos une promesse de répétition des échanges inscrite dans le long terme. Le credito est alors défini comme une reconnaissance de dette, un bon à valoir sur une quantité de travail que le troqueur devra fournir au groupe. Les deux partenaires sont quittes l'un

10 Avant de participer à leur première foire, les nouveaux membres du « nœud de récupération » devaient suivre les cours d'introduction au troc afin d'être initiés à l'éthique du Réseau Global de Troc. 
envers l'autre, mais ils restent liés par leur engagement envers la communauté en raison de l'inaliénabilité du credito qui, en théorie du moins, relève toujours de la propriété du réseau. La fermeture d'un troc en ouvre toujours un autre, l'acquittement de la dette par la remise des creditos induisant simultanément la création d'une nouvelle dette.

Les fondateurs estiment que le caractère « immortel » de la monnaie induit un processus d'accumulation, lui-même vecteur d'inégalité. Cela les amène, en fin de parcours, à doter le credito d'un nouvel attribut destiné à en faire une monnaie « naturelle ». Dans la nature, tout se décompose, aussi, fidèles à leurs convictions écologiques, ont-ils décidé d'ôter à leur moyen d'échange cette propriété « surnaturelle » qu'est l'immortalité en mettant en place un système d' « oxydation ». Il s'agit d'une taxe sur l'accumulation (1\% par mois), qui prend le contrepied du système d'intérêts du capitalisme. L'objectif est de stimuler la circulation des creditos et de favoriser de cette manière la production et la consommation. Le credito reprend ainsi le flambeau de diverses expériences européennes de monnaie fondante également inspirées de la théorie d'Alvin Toffler (1980).

Une fois établi, l'usage du credito devient obligatoire au sein du réseau, car les prosommateurs qui "n'utilisent pas notre monnaie, [...] n'appartiennent pas à notre groupe »(Guillermo Magadan). L'utilisation de cette monnaie interne traduit bien l'intégration au groupe et la promesse d'en respecter les douze principes de fonctionnement.

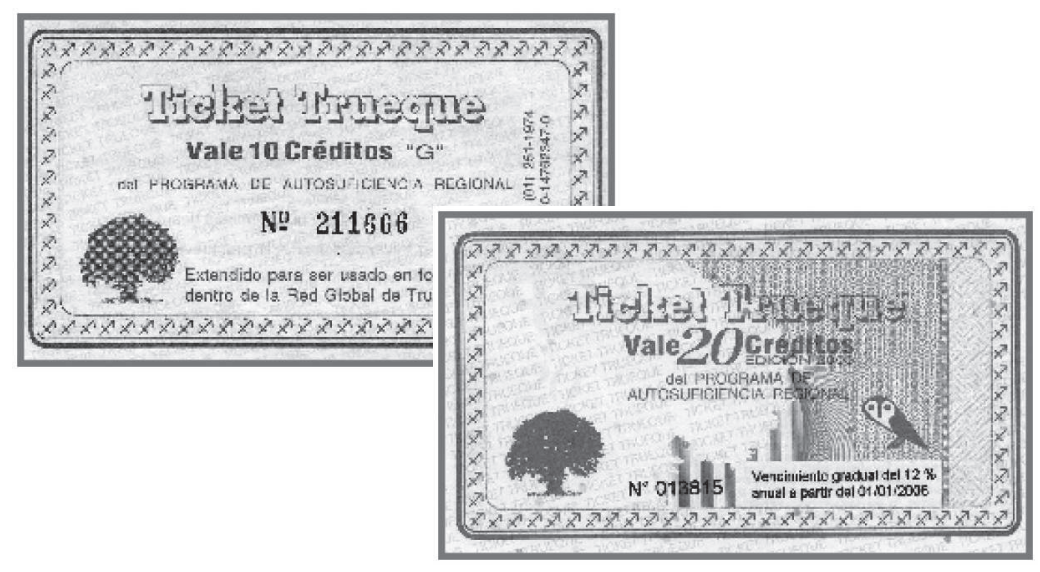

Derrière ce discours qui se veut révolutionnaire, ce que proposent les fondateurs, ce sont des «petits papiers » imprimés par leurs soins qui fonctionneraient comme une monnaie dans la communauté des prosommateurs. Il conviendrait certainement de se demander si le credito est aussi différent de l'argent que ses créateurs veulent nous le faire entendre. S'il s'agit d'une monnaie particulière, à quel genre de monnaie avonsnous affaire? Si elle revêt l'apparence d'un papier-monnaie aux allures modernes, ne partage-t-elle pas également certains points communs avec les monnaies que nous avons coutume de qualifier de « traditionnelles $»^{11}$ ? Comme nous ne souhaitons pas entrer dans

11 A ce sujet, voir notement Jonathan Parry et Maurice Bloch (1993), Jean-Michel Servet (1998) et Alain Testart (2001). 
ce débat qui nous mènerait au-delà du thème traité dans cet article, nous nous bornerons à soulever ces questions qui mériteraient d'être traitées avec beaucoup d'attention. En effet, comme la description ethnographique le montrera, le credito est un instrument monétaire mouvant qui ne peut être défini ni par ses fonctions (potentielles ou effectives), ni par la forme des prestations dans lesquelles il intervient, ni par la nature de la relation qui unit les partenaires de la transaction. Pour pouvoir embrasser les multiples figures conférées au credito par ses utilisateurs, il est donc nécessaire de s'en tenir à une définition large du phénomène monétaire. C'est la position adoptée par Maurice Godelier, qui considère comme une monnaie tout bien qui constitue « un équivalent général, un intermédiaire obligatoire pour accéder à toutes les marchandises socialement disponibles et nécessaires » (1969 : 27). C'est effectivement ce rôle qu'est amené à jouer le credito dans la sphère du trueque.

Sachant que l'absence de recours à un médiateur d'échange constitue l'une des caractéristiques fondamentales du troc, l'introduction du credito dans ce système économique nous invite à nous interroger sur la pertinence de la catégorie de troc dans le cas présent. Si ce n'était pas le troc, quelle modalité d'échange économique les pionniers du réseau entendaient-ils mettre à l'honneur ? Si le trueque recourt à la monnaie, comme le fait l'échange marchand en pesos prépondérant dans l'économie formelle, il s'en distingue néanmoins par un certain nombre de points fondamentaux. Alors que ces échanges marchands sont de nature accumulatrice, aucun profit matériel ne doit être engendré par le trueque. Contrairement à ce que l'on entend communément par « marchandise », les biens du trueque, pour autant qu'ils soient produits et échangés dans les règles de l'art, conservent toujours l'empreinte de leur confectionneur. En tant que surplus de la production domestique, ce qui circule dans le nodo est de même nature que ce qui est consommé au sein du foyer. A ce titre, ces produits portent une part de l'intimité de leur producteur, qui est transmise aux consommateurs à travers les échanges ${ }^{12}$. Nous adopterons dans cet article le point de vue d'Igor Kopytoff (1986), qui considère les biens comme présentant, à chaque étape de leur parcours, une position spécifique dans le processus de marchandisation. A la lumière de cette grille d'analyse, les biens évoluant dans la sphère du trueque devraient revêtir un plus grand degré de singularité que les marchandises qui évoluent dans le marché formel. En effet, cet auteur propose d'envisager comme marchandise toute « chose qui a une valeur d'usage et qui peut être échangée pour une contrepartie dans une transaction discrète » (1986:68, notre traduction); tandis que les biens de trueque sont destinés à intervenir dans des prestations engageant les partenaires à une réciprocité pérenne.

Il nous semble que le trueque idéal s'apparente à un mode d'échange que l'on pourrait qualifier d' " oblatif », au sens définit par Marcel Mauss, à savoir, un geste « apparemment libre et gratuit, et cependant contraint et intéressé » 2004 :147). Ainsi, dans le monde conceptuel du trueque, les facettes « intéressée » et « altruiste » de l'activité économique se manifesteraient simultanément dans un seul mode d'échange, et si l'intégration dans le nodo est entièrement démocratique et relève de l'initiative de l'adhérent, une fois associé, celui-ci ne pourra pas faillir à son engagement de reproduire les échanges sur le

12 Comme le suggère Alfred Gell, nous considérerons que l'inaliénabilité, dans ce cas, ne porte pas sur les biens qui changent effectivement de propriétaire mais plutôt sur «l'identité sociale du donateur qui reste attachée à l'objet après que celui-ci a été donné » (1992:145, notre traduction). 
long terme sans subir l'opprobre collectif. Par ailleurs, les transactions réalisées dans le respect des principes définis par les fondateurs reposent sur des considérations embrassant l'ensemble des champs sociaux; les dimensions éthique, écologique ou festive sont explicitement mentionnées. Ainsi, dans leur conception idéale, tout se passe comme si ces échanges devaient se réaliser sous la forme de " prestations sociales totales ». Ce concept introduit par Marcel Mauss renvoie à un mode de circulation des biens qui met en branle « toutes sortes d'institutions » (Mauss, 2004 : 147) du groupe social en question. Dans les paragraphes suivants, nous présenterons deux exemples de mise en pratique du système socioéconomique élaboré par les fondateurs, et nous tenterons de comprendre à quelles formes d'échange renvoie le trueque tel qu'il est pratiqué par les acteurs.

\section{Les clubs Color Esperanza et Bernalecita : deux exemples contrastés}

Il n'existe pas de modèle unique d'organisation dans le réseau: chaque nodo se caractérise par une division du travail et des relations sociales spécifiques, par les logiques qui sous-tendent les échanges économiques et par la quantité et la qualité des biens symboliques et matériels en circulation. Ils disposent d'une organisation politique propre et se déroulent dans des structures spatio-temporelles particulières. En théorie, l'acceptation des douze principes du réseau conditionne l'intégration des adhérents. En réalité, le modèle initial a donné lieu à une multitude d'interprétations plus ou moins conformes aux valeurs originelles. Les pages suivantes seront consacrées à une présentation plus détaillées de deux clubs : Color Esperanza et Bernalecita. Ce choix se justifie par la place qu'ils occupent aux deux extrêmes d'un continuum : le premier est considéré par les fondateurs comme une interprétation idéale de leurs principes, tandis que le second constitue ce que l'on pourrait appeler un « club parasite », revendiquant son appartenance au réseau sans manifester le souci d'en respecter l'idéologie. Je conclurai en situant ces cas particuliers dans une réalité plus large : celle du réseau dans son ensemble. L'enquête s'est déroulée en 2003, et j'emploie ici le présent ethnographique.

\section{Color Esperanza}

Les membres du club Color Esperanza se réunissent chez la coordinatrice, Alejandra. Dans son garage sont exposés les divers produits, tandis que son salon sert d'entrepôt et son jardin, de « salle d'attente ». C'est dans ce club que j'ai observé l'investissement symbolique et affectif de l'espace le plus prononcé. Sur les portes, les armoires et les cloisons sont affichés une multitude de messages: les principes éthiques du réseau, des petites annonces, des célébrations de fêtes, etc. Nous pouvons lire, par exemple : « Ici nous troquons ton désespoir pour de l'espérance, ta lutte solitaire pour une union solidaire avec des gens travailleurs qui construisent une société meilleure jour après jour ». Un drapeau national est suspendu au-dessus des étagères, et un tableau est laissé à la disposition des participants pour y noter les services qu'ils proposent. Les foires du nœud Color Esperanza ont lieu quatre fois par semaine, afin de permettre aux troqueurs de s'approvisionner en nourriture fraîche de manière régulière.

Le jardin d'Alejandra, qui sert de salle d'attente, est un lieu propice aux bavardages. Les prosommateurs s'y donnent des conseils, échangent des expériences et commentent l'actualité nationale.

Une foire dure entre trois et quatre heures et se déroule en plusieurs étapes. Après avoir payé leur droit d'entrée (un peso), les participants déposent leurs produits sur une 
table et reçoivent en échange un numéro d'ordre et un papier où figure le total de la valeur en creditos des denrées. Ensuite, un tirage au sort détermine l'ordre de passage devant la table. En attendant leur tour, les troqueurs discutent et partagent le maté, qui est habituellement accompagné de beignets offerts par le club.

Le nombre de participants varie selon les jours. Les mardi, mercredi et jeudi, il est limité à une trentaine, alors que les samedis, les troqueurs se font plus nombreux, atteignant entre soixante et soixante-dix participants. Cette différence est due à la présence des ouvriers de La Perla, une usine de pains sauvée de la faillite par l'établissement d'un partenariat. Les ouvriers reçoivent une partie de leur salaire en creditos et Alejandra reçoit la contrevaleur en pains, qu'elle troque à son tour pour une quantité de creditos supérieure.

Les membres habituels vivent aux alentours du club, rares sont ceux qui sont motorisés. Certains viennent du bidonville situé à proximité. La majorité sont des femmes sans emploi. Certaines sont à la retraite, mais ne peuvent survivre avec les maigres allocations qu'elles reçoivent. D'autres se sont intégrées au groupe lorsqu'elles, leur mari ou les deux, ont perdu leur emploi. D'autres encore sont venues par curiosité, accompagnant une amie ou une voisine, et sont ensuite restées, estimant que le troc leur convenait. Il y a également plusieurs participantes dont la situation économique s'est améliorée, mais qui continuent à venir par plaisir.

L'essentiel de l'organisation est pris en charge par la charismatique Alejandra. Créative et dynamique, elle trouve toujours une solution à tout. Lors des foires, elle est aidée par ses enfants et des amies également impliquées dans le réseau. Bien qu'elle soit encouragée, la participation des autres prosommateurs est restreinte. Ils s'accordent pourtant pour affirmer le caractère démocratique de l'organisation. Alejandra aimerait que ses partenaires prennent leur indépendance, mais ils ne le font pas : "Certains sont plus participatifs que d'autres. De temps en temps ils proposent une idée, mais c'est rare. Premièrement, ils manquent de confiance en eux et ensuite, c'est plus commode de ne pas se compromettre ". Mais si certains ne se manifestent pas, c'est aussi parce qu'ils sont convaincus que l'organisation relève des fonctions de la coordinatrice. Selon Gloria (57 ans, sans emploi) : " Alejandra est responsable de son nodo, c'est-à-dire d'organiser et de chercher des solutions pour nous ».

[photo 2]

L'autorité incombe également à Alejandra. Ce rôle est essentiel parce que, dit-elle : " il faut tout le temps sanctionner ». J'ai plusieurs fois assisté au rappel à l'ordre des troqueurs indisciplinés : ceux qui ne consomment et ne produisent pas en quantités équivalentes, ceux qui ne fournissent pas assez de biens comportant une valeur ajoutée, ceux qui exigent des portions supérieures à celles qui leur reviennent, etc. Ce manque de discipline s'explique en partie par le fait que tous n'ont pas assimilé les douze principes du troc. La plupart en ont pris connaissance par l'intermédiaire d'Alejandra et estiment qu'ils sont efficaces. Cependant, lorsque je les ai interrogés sur leur contenu, les réponses étaient moins précises : "Les douze principes? Je ne les ai jamais lus. Ah! Si, je les ai lus, mais je ne m'en souviens plus ", me confie Gloria.

Les biens proposés sont essentiellement des produits alimentaires. La majorité des prosommateurs viennent avec des plats préparés: hamburgers de soja, pâtisseries, beignets, pains, etc. Généralement, une partie est consommée directement au sein du foyer et l'autre est réservée à l'échange. Grâce au peso de participation, Alejandra se procure la farine, le sucre et le soja en gros, et les redistribue en échange de creditos. 
Ceux qui les utilisent pour cuisiner ont priorité. L'huile ne circule pas, ce qui force les participants à bénéficier d'une source minimale de revenu en pesos s'ils veulent continuer à participer aux échanges. Une partie des pains de La Perla est échangée pour des légumes frais. Les épices et les produits ménagers sont généralement achetés en gros en pesos et écoulés au détail en creditos. L'artisanat est également représenté, mais en faible proportion. Quelques services sont proposés : coiffeur, week-end à la campagne, massages..., ainsi que différents cours : informatique, langues, crochet, tissage, etc. Des produits usagés sont également échangés; il s'agit essentiellement de vêtements. Leur circulation est plus faible que celle des produits alimentaires, car Alejandra exige que l'on apporte régulièrement des produits renfermant de la valeur ajoutée. Les denrées sont nombreuses et variées. En suscitant la collaboration entre ses voisins, Alejandra fait apparaître l'abondance dans un espace en pénurie. Il n'est pas rare que des produits périssables soient offerts en fin de foire, afin qu'ils ne soient pas gaspillés.

Au nœud Color Esperanza, c'est Alejandra qui détermine la valeur d'échange. Elle a attribué au credito une valeur fictive de cinquante centavos. Cette équivalence lui sert de repère pour établir des prix proportionnels à ceux du marché formel : «Si c'est un produit élaboré, j'ajoute les frais qu'aurait induits sa production dans le commerce. C'est là que réside le gain du producteur, puisqu'il n'a pas de frais d'entreprise ». La valeur des produits plus rares est évaluée en accord avec le producteur. Je n'ai assisté à aucun litige à ce propos. Remarquons que la loi de l'offre et de la demande est totalement absente de la formation des prix, il arrive régulièrement que la nourriture se périme sans qu'aucun remboursement ne soit exigé auprès de celui qui a apporté le produit dont personne n'a voulu.

Lors des foires, les creditos ne circulent jamais directement entre les partenaires ; les marchandises transitent par la communauté avant d'être redistribuées au prorata des valeurs produites par chacun. Les prosommateurs sont vivement encouragés à consommer en quantité équivalente à leur production. Cependant, la stricte égalité est très rarement atteinte et les creditos permettent d'équilibrer la transaction. Alejandra effectue ainsi une centralisation des comptes pour l'ensemble du nœud. Elle gère tous les transferts à l'exception de ceux qui se font de manière informelle et ont généralement lieu sans creditos. Au cours de l'échange, les biens peuvent se doter d'une plus-value symbolique, à la valeur de l'objet s'ajoutera celle du lien qui unit les partenaires entre eux, ou à la communauté, cette valeur pouvant s'intensifier dans l'échange. La valeur totale d'un objet est donc fonction de sa valeur d'échange, mais également de sa capacité à agir sur la santé sociale des protagonistes.

Dans ce club, les principales valeurs sont celles des fondateurs du Réseau : confiance, solidarité et intégration. Les manifestations de suspicion et de concurrence, comme la recherche de profit personnel sont présentes chez certains prosommateurs, mais elles sont réprimées dans les comportements. Dans ce trueque, ce qui est en mis en jeu n'est pas uniquement la valeur des biens, mais également celle de son producteur. En révélant aux exclus de l'économie formelle leur capacité à produire au moins en partie les biens dont ils ont besoin pour vivre, le troc leur permet de retrouver une certaine dignité. Enfin, la participation aux échanges peut éveiller chez les citoyens esseulés un sentiment d'appartenance à une collectivité. Au sein du nœud vont se créer de nouvelles amitiés. L'affection et la compagnie sont valorisées par les prosommateurs heureux d'avoir découvert un nouveau milieu convivial qui leur offre par ailleurs l'occasion de se constituer un carnet d'adresse utile en cas de coup dur. Pour Alicia : " Chaque jour, tu 
trouves quelque chose de matériel et de spirituel. Chaque jour, tu reçois un soutien. En tendant l'oreille, tu découvres des valeurs chez les gens parce qu'à part la misère, il y a autre chose. Pas uniquement le matériel mais aussi ton temps ou ton écoute ».

Au Color Esperanza, l'échange doit se montrer désintéressé, même si pour certains, « il n’y a que fiction, formalisme et mensonge social » (Mauss, 2004 : 147). Par ailleurs, l'accès au club est libre, mais une fois que les échanges sont engagés, la participation régulière de chacun devient impérative, sous peine de se voir exclu de la collectivité. Une autre particularité des prestations sociales totales analysées par Mauss est également observable dans le trueque du Color Esperanza, puisque les personnes et leurs biens ne sont jamais complètement distincts. Les biens témoignent de la valeur de leur producteur, dont ils conservent l'empreinte, même après la transaction. Ainsi, un compagnon me mettra en garde : "Il faut se méfier de ces plats-là. Celle qui les prépare vient du bidonville. Là-bas en face. Il n'y a pas de frigo. Et les cafards grouillent... " (George, 27 ans, ouvrier à La Perla). Si l'origine des prosommateurs est tue par Alejandra qui entend éviter ce genre de discrimination, les biens que cette femme produit (essentiellement de la polenta, qui, à l'égal du soya, remplace la viande pour les familles qui n'y ont pas accès) révèlent son identité. Guillermo aimait pour sa part apporter des conserves d'aubergines marinées tout à fait raffinées, produisant une autre image personnelle et suscitant l'admiration générale. Les biens mis en circulation dans ce trueque reflètent ainsi le statut de leur producteur, lui assignant de fait un rang dans la hiérarchie sociale du nodo. Ces échanges monétaires partagent, de ce point de vue, une identité structurelle avec le troc que Hugh-Jones et Humphrey envisagent comme un « échange total, dans lequel les gens sont identifiés aux produits de leur mode de vie et vice-versa » (1992:10, notre traduction).

\section{La Bernalecita}

Les foires de la Bernalecita prennent place dans un sinistre hangar. L'obscurité y est pesante, car l'unique porte ne suffit pas à éclairer cet endroit, qui n'est pas approvisionné en électricité. Une entrée très étroite permet de contrôler l'arrivée des participants et de récolter leur droit d'entrée (un peso). L'espace est rempli par les tables chargées de marchandises. L'allée qui sépare l'entrepôt de la chaussée est envahie par ceux qui viennent vendre des produits en pesos. Ne pouvant entrer car la circulation des pesos est interdite à l'intérieur du hangar, ils constituent un marché parallèle.

A la Bernalecita, les réunions se déroulent quatre fois par semaine en fin d'aprèsmidi. Dès l'ouverture des portes, tous se dépêchent d'installer leurs affaires afin de pouvoir accéder le plus vite possible aux denrées prisées. Le stress envahit l'atmosphère : "Parfois, tu viens et puis tu commences à devenir nerveuse parce que tu ne trouves rien " (Christina, 46 ans, troqueuse sans emploi). C'est l'obscurité qui met fin aux échanges.

Le nombre de participants varie selon les jours. Les mercredi et samedi, il quadruple, atteignant deux cents participants. Cette augmentation est due à la présence de producteurs motorisés venant des communes alentour. Ils apportent des produits frais et attirent par là des troqueurs ponctuels. Ces jours-là, l'ambiance est impersonnelle, car les participants, qui viennent parfois de loin, ne se connaissent pas. Lors des foires calmes qui ne rassemblent pas plus qu'une cinquantaine de personnes, l'ambiance est plus intime. Les participants sont les gens du quartier, dont la grande majorité n'est pas motorisée. Ce sont des habitués, qui viennent autant pour troquer que "pour se distraire », comme le souligne Christina, responsable du nettoyage : "Parce que rester toute la journée à la maison... en plus avec mon mari qui est insupportable parce qu'il n'a pas de travail!"». 
La composition sociale de la Bernalecita est très hétérogène et varie selon les jours. " Ici, il y a de tout. Des hommes, des femmes, des jeunes et beaucoup de personnes âgées pour qui la retraite est insuffisante " (Christina).

Les participants sont unanimes à critiquer l'organisation du nodo. Si la situation ne s'améliore pas, c'est parce que sa structure politique les décourage de s'investir davantage: "Moi, je ne peux rien faire parce qu'ici il y a des dirigeants; moi je n'ai rien à voir avec le sommet. Je suis une troqueuse de plus et c'est tout! " affirme Paola, 52 ans, une troqueuse sans emploi, également responsable du nettoyage. Les discours portant sur l'autorité présentent des divergences étonnantes témoignant du désaccord existant entre les participants. Ainsi, le coordinateur estime que le mécanisme de prise de décision est « totalement démocratique ». Paola affirme quant à elle que « celui qui prend les décisions, c'est le coordinateur ». Elle soutient également que les dysfonctionnements du club sont dus aux "gens de l'extérieur ». "Entre nous, on se connaît et on se fait confiance. Ce qui se passe, c'est que ceux qui ont la marchandise, ils te disent 'oui, oui', et puis ils continuent à troquer de la même manière ». Ce que Paola dénonce, c'est le pouvoir qu'exercent les détenteurs des produits frais, indispensables au fonctionnement du club. Maria, 61 ans, tient un discours accusateur et résigné en relatant une escroquerie dont elle a été victime : "En réalité, c'était un ami du coordinateur. Ils se connaissent et ils s'arrangent entre eux. Moi je me suis habituée à me défendre seule parce que si tu dois attendre que les autres le fassent... Je fais semblant de rien. Ici, c'est la loi de la jungle ».

La quantité et la variété des produits en circulation dépendent de la fréquentation et des jours de réunion. Les mercredi et samedi et principalement pendant le premier quart d'heure, il est possible de se procurer des œufs, de la viande, des fruits ou des légumes, totalement absents les autres jours. Les denrées de base qui sont présentes en moindre quantité lorsque manquent les produits frais, sont généralement achetées sur le marché formel et ensuite échangées dans le club contre une quantité de creditos permettant de s'offrir certaines denrées en quantité supérieure à ce qu'il est possible d'acheter en pesos. Pour effectuer ces transactions spéculatives, il faut bénéficier d'un revenu en pesos, ce qui n'est pas le cas de tout le monde. D'autres viennent écouler les marchandises qu'ils reçoivent parfois en guise de salaire. Des mères viennent également échanger une partie des aliments fournis par l'Etat ${ }^{13}$ contre du frais. Mentionnons encore les objets de seconde main, qui constituent la majorité des échanges les jours où il n'y a pas de produits frais. N'importe quel objet peut être intégré au circuit. Tout est question de chance, il faut tomber sur « la bonne affaire»: appareils photos, vêtements, ustensiles ménagers ou encore outils de bricolage. Enfin, nous observons la présence de marchandises volées, dont le statut est généralement connu de tous sans que cela ne gêne les transactions.

A la Bernalecita, des phases de parité fictive avec le peso alternent avec des phases de "libération» du cours du credito. Dans un cas comme dans l'autre, ce sont les prosommateurs qui définissent en dernière instance la valeur des denrées qu'ils entendent échanger. Comme le dit le coordinateur, "chacun se charge de son kiosque ».

Lorsque la parité est observée, la liberté est limitée par l'équivalence approximative avec le marché formel. Cependant, certains détenteurs de biens rares profitent de leur

13 Certaines mères d'enfants en bas âge bénéficient du Plan vida. Il s'agit d'une aide alimentaire en produits de base (farine, riz, pâtes ou lait) distribuée mensuellement. 
quasi-monopole pour échanger leurs produits " par-dessous les tables » afin d'échapper au principe de parité sans éveiller la colère de leurs partenaires. Les participants feignent d'ignorer ces pratiques pour éviter les conflits et se donner la possibilité de braver l'interdit à leur tour. Pendant ces périodes, la valeur du credito est relativement stable, mais le volume des échanges est restreint, car les fournisseurs en produits frais se retirent du circuit. Disposant d'un moyen de locomotion, ils préfèrent se rendre dans d'autres clubs où ils pourront obtenir davantage de creditos. Les pourvoyeurs de produits de base, dont la participation est généralement motivée par la présence de produits frais, se feront aussi plus rares. Petit à petit, les prosommateurs vont déserter le club qui s'écartera toujours plus de leurs attentes. La libération des prix sera alors envisagée comme l'unique solution. Cette mesure donne le feu vert aux pratiques inflationnistes. La loi de l'offre et de la demande règne alors en maître absolu. Les ravitailleurs en produits frais confèrent une valeur élevée à leur marchandise, car ils savent que leur présence est indispensable au bon fonctionnement du club. Ils seront suivis par les détenteurs des produits de base. L'inflation se généralise rapidement, et au cours d'une même foire les prix grimpent à une vitesse telle que certains produits circulent de main en main pour une valeur chaque fois supérieure. La valeur d'échange échappe à toute régulation. Elle dépendra des relations interpersonnelles, de la capacité de persuasion des partenaires et des stratégies qu'ils élaborent. Certains préfèreront vendre moins cher, en plus grande quantité et plus vite, tandis que d'autres maintiendront des prix abusifs, attendant la fin de la foire pour faire jouer la loi de l'offre et de la demande en leur faveur. La flambée des prix s'accompagne d'une redéfinition implicite de la valeur des creditos à l'intérieur du club. Les quantités nécessaires aux transactions sont en effet tellement importantes ${ }^{14}$ qu'il est impossible de contrôler les billets contenus dans la liasse en transit. Ils se verront alors attribuer une valeur unique de cinquante creditos, quel que soit le montant indiqué sur le papier. Les bons inférieurs à dix creditos étant hors d'usage en période de « libération », la confusion n'aura lieu qu'entre les billets de vingt et de cinquante creditos. Cette « règle » est jugée anormale par les participants, qui ne manquent pourtant pas de l'appliquer. Il est ainsi admis que l'on puisse glisser des billets de vingt creditos dans une liasse de billets de cinquante, tandis que réaliser la même opération avec des billets de dix serait malhonnête. Il est également intéressant de noter l'abondance de billets falsifiés. Ils sont reconnus comme tels par leurs utilisateurs, qui ne voient cependant pas de bonne raison de s'en séparer. "C'est aussi des creditos! Non ? " répondait Paola aux questions portant sur leur validité.

Lorsque l'inflation deviendra incontrôlable et que même les billets falsifiés ne suffiront plus pour constituer les liasses nécessaires aux transactions, les habitués menaceront à leur tour de déserter, laissant les détenteurs des produits frais sans acheteurs, et l'arrimage au peso sera à nouveau imposé jusqu'aux prochaines prises de mesures par le coordinateur.

Il apparaît avec évidence que dans ce nodo, les valeurs originelles du trueque ne sont pas reconnues. Voici ce qu'en dit le coordinateur : "L'esprit est individualiste. De la solidarité? Non, ça, ça n'a jamais existé. [...] Ici, c'est du libre commerce ». Les fondateurs connaissent l'existence de ce club qui, disent-ils, souille leur image. Ils dénient au coordinateur le droit de revendiquer son appartenance au réseau, sans avoir

14 Lors de nos observations, un litre d'huile pouvait valoir, par exemple, environ 30000 creditos. 
pour autant entrepris la moindre démarche pour l'exclure. Les valeurs du réseau peuvent être toutefois respectées dans le cadre de relations individuelles entre habitués. Ces derniers, qui constituent de rares exceptions, entretiennent des liens riches de confiance ou de solidarité, qui ne seront jamais élargis à l'ensemble du groupe. Pour la plupart des membres, le troc constitue une économie de seconde zone à laquelle ils s'intègrent faute de mieux. La participation aux échanges, qui apparait comme le symbole de leur paupérisation, est alors source de honte.

Par contraste avec ce que nous avons observé dans le cas du Color Esperanza, les foires de la Bernalecita constituent une sphère d'échange soumise en grande partie à l'ordre marchand. Pour que puisse s'instaurer un tel ordre, Louis Dumont remarque qu'il faut que les relations aux choses puissent s'instituer indépendamment des relations aux personnes ${ }^{15}$. C'est effectivement le cas à la Bernalecita : les biens qui circulent en son sein sont généralement produits ou achetés pour être revendus, et sont de ce fait pleinement dissociables du vendeur, et, de plus, les liens qui s'instaurent entre prosommateurs anonymes se limitent généralement au temps de la transaction économique. Au gré des rencontres, les partenaires définissent les modalités de leurs transactions, la maximisation du profit étant toujours la considération prépondérante. Dans ce contexte, le credito fait l'objet de représentations qui l'apparentent au peso. Il est d'ailleurs généralement nommé "plata (argent) par ses détenteurs.

\section{D’une manière générale}

Toute tentative d'inscrire le trueque dans une modalité d'échange spécifique serait abusive. Il est nécessaire d'envisager chaque club comme une appropriation spécifique du modèle des fondateurs. Il faut également tenir compte des représentations mobilisées par les membres, tout en sachant qu'elles s'expriment de manière plus ou moins franche selon l'espace de liberté offert par l'organisation du club. Néanmoins, les observations réalisées dans ces différents clubs de la banlieue de Buenos Aires nous permettent de repérer les deux extrémités d'un continuum dans lequel se retrouvent, entrelacées selon des modalités propres à chaque nodo, les caractéristiques décrites ci-dessous.

D'un côté, nous observons des clubs fortement intégrés dans lesquels les membres veillent au bon respect de «l'esprit du troc ». Les liens primaires qui se nouent dans ces clubs sont placés sous le signe d'une solidarité et d'une confiance accrues. Dans certains cas, les relations sociales inscrites dans la durée l'emportent sur les gains économiques. Pour faire valoir les liens tissés, des règles plus ou moins explicites circonscrivent le modèle de transaction. Chacun sait qu'il est inconvenant de marchander, d'exiger, d'accaparer. Les valeurs sont définies dans un climat amical : l'essentiel, c'est l'échange et non le profit matériel. Le credito fait alors l'objet de représentations spécifiques qui encouragent les troqueurs à en faire un usage qui respecte les valeurs économiques, sociales et politiques affichées par le réseau. Les biens qui circulent sont personnalisés puisqu'ils sont identifiés à leur producteur, dont ils gardent l'empreinte après avoir changé de mains. Loin de constituer des échanges exclusivement économiques, ces transferts peuvent être qualifiés de prestations sociales totales.

Cité par Caillé (2005:183). 
D'un autre côté, nous trouvons les clubs que nous avons qualifiés de "parasites ", dont les membres ne partagent pas une éthique commune. Le profit et l'intérêt matériel constituent leur motivation fondamentale, et des échanges économiques ponctuels constituent leur unique point de rencontre. Le credito, dont l'usage est alors semblable à celui du peso, ne fait plus l'objet des représentations spécifiques qui découragent les pratiques de spéculation et d'accumulation. Les biens en circulation présentent un degré de marchandisation accru pour reprendre à nouveau les termes de Kopytoff (1986). Les transferts réalisés sur le mode de l'échange marchand ne font l'objet d'aucune restriction politique ou sociale, si bien que la ruse, le vol et l'escroquerie deviennent des pratiques courantes dans ces nodos, qui ont tendance à reproduire ce contre quoi le troc s'était proposé de lutter.

Evidemment, ces deux modèles se présentent de manière plus nuancée dans la réalité. Ainsi, dans un club donné, tous les membres ne sont pas animés des mêmes intentions. En effet, j'ai rencontré à la Bernalecita, qui relèverait plutôt du second cas de figure, des participants qui ont développé des complicités allant au-delà de leurs intérêts économiques et dont les relations s'apparentent à celles qui unissent les individus de l'idéal type opposé. Ceci confirme que les échanges de type marchand, lorsqu'ils sont répétés, peuvent également servir d'assise à des relations sociales pérennes. Inversement, j'ai rencontré des «prosommateurs parasites » dans des clubs où la cohésion est forte. Ainsi, au club Color Esperanza, même si tous pratiquent les échanges selon les normes en vigueur, certains ne respectent les règles que sous le poids de la contrainte sans être convaincus de leur bien fondé. La même transaction peut être envisagée sous un angle différent de la part de chacun des protagonistes. Ce fait, qui mérite d'être souligné, doit nous inciter à d'autant plus de prudence dans l'analyse des comportements économiques. $\mathrm{Au}$ Color Esperanza, si tous les prosommateurs réalisent à première vue le même type de prestation, le discours révèle un écart significatif dans les représentations : le trueque y est toujours défini comme un échange, mais pour certains membres il se distingue de l'échange marchand, tandis que pour d'autres, il semble s'y assimiler. Par exemple, Suzi l'envisage comme "l'échange de ce que quelqu'un fait contre quelque chose dont il a réellement besoin ", alors que pour Gloria, troquer, c'est réaliser un échange dans un nœud qui n'est autre qu' "un magasin où l'on peut aller acheter différentes marchandises». Cela nous incite à affirmer que ce qui distingue les différentes figures d'échanges relève en dernier ressort de l'ordre cognitif.

En chargeant leurs «petits papiers » de représentations précises, les fondateurs espéraient en cantonner l'usage à une modalité d'échange spécifique, mais les prosommateurs se sont approprié le moyen d'échange qui leur était proposé pour le mettre à profit selon leur convenance. Nous y voyons une nouvelle confirmation de la capacité des individus à absorber « les monnaies, les transformant pour les adapter à une riche gamme de valeurs et de relations sociales » (Zelizer 2005 :13). Le credito en est ainsi venu à revêtir des rôles spécifiques selon les partenaires en jeu et l'environnement socioéconomique des échanges. Nous rejoignons les auteurs qui, se démarquant du fameux texte de Simmel (1999), affirment que ce n'est pas la monnaie qui est responsable de la dissolution des liens sociaux, mais bien les usages qui en sont faits, lesquels sont eux-mêmes indissociables de la matrice culturelle dont ils émergent. 


\section{Conclusion}

En adoptant un point de vue un peu sévère sur ce mouvement, nous pourrions commencer par remarquer qu'en dépit de leurs discours révolutionnaires, les fondateurs n'ont finalement pas fait autre chose que réinventer la monnaie. Mais il faut nuancer, en effet, l'innovation ne portait pas tant sur la nature de cette « invention » que sur la possibilité même, pour ces citoyens post-modernes, de mettre en circulation un instrument monétaire à portée nationale dont ils contrôlaient seuls l'émission. A mesure que les prosommateurs l'identifiaient à l'argent et l'utilisaient comme tel, les pionniers se sont efforcés de doter leur instrument monétaire de propriétés qui devaient le singulariser. Plus que l'objet matériel lui-même, ce sont les représentations dont était investie cette « monnaie sociale » qui devaient se révéler novatrices. C'est lorsque le credito se trouva effectivement pourvu d'un imaginaire économique qui lui appartenait en propre que le trueque put constituer un terrain propice à la reconstitution tout à la fois matérielle et symbolique de la classe moyenne déchue. Il devait alors, d'une part, satisfaire les besoins de subsistance des citoyens appauvris et, d'autre part, recréer un système économique dans lequel les pratiques de production, d'échange et de consommation pouvaient renvoyer à une part de leur identité, comme c'était le cas dans le système capitaliste avant qu'il ne soit frappé par la crise. Lorsque la cohésion sociale fondée sur le partage d'une identité commune contribuait à garantir le caractère éthique des activités économiques, les multiples figures de l'échange qui se sont déployées autour du credito ont pu constituer les vecteurs signifiants d'une « intimité culturelle » (Herzfeld 2005) naissante témoignant de la reconstruction sociale des citoyens. En revanche, lorsque la maximisation du profit constituait le mobile principal des prestations, le trueque non balisé par la loi devenait un espace propice au déploiement de stratégies opportunistes, et les échanges auxquels il donnait lieu ne faisaient que confirmer publiquement le déclassement des prestataires en soulignant leur intégration dans une «économie du pauvre». La reconstruction symbolique de cette classe désagrégée s'est ainsi opérée non seulement par l'adoption d'un style de production et de consommation (artisanal, « du terroir », biologique, etc.) destiné à mettre en valeur les ressources locales dépréciées sur le marché national, mais aussi par la création de figures d'échanges qui devaient se démarquer du système formel.

Ce que proposa le trueque aux déclassés, avant qu'iln'entre lui-même en crise, c'est donc un espace dans lequel les échanges économiques (en tant que maillon partagé socialement d'un système plus large qui s'y trouve symbolisé) révélaient une intimité culturelle faisant idéalement l'objet d'une revendication identitaire. Les biens en circulation, loin d'être des produits de seconde zone, purent dès lors apparaître comme autant de symboles des valeurs remises à l'honneur par les déclassés, qui se démarquaient du système capitaliste accusé d'avoir rongé leur société. Dans ce cas, les prestations économiques purent être elles-mêmes envisagées comme un mode d'expression dont se seraient appropriés les acteurs pour donner consistance à leur imaginaire socioéconomique. Les biens matériels mis en circulation, en synchronie avec les flots de paroles qui les accompagnaient (sous la forme de discours révolutionnaires stéréotypés) se sont mutuellement soutenus dans ce processus de recomposition identitaire. Dans le cas du trueque, c'est par le truchement des comportements économiques que la classe moyenne argentine put instaurer des pratiques signifiantes dans un environnement social dévasté par la crise. 


\section{Références bibliographiques}

Bloch, Maurice et Jonathan PARry, 1993 [1989]. «Introduction: Money and the Morality of Exchange », in Maurice Bloch, Jonathan Parry (éds.), Money and the Morality of Exchange, pp. 1-32. Cambridge, Cambridge University Press.

Bourdieu, Pierre, 1979. La distinction, critique sociale du jugement, Paris, Les Editions de Minuit.

Covas, Horacio, Carlos De SAnzo et Ruben Ravera, 1998. Reinventando el mercado : la experiencia de la Red Global de Trueque en Argentina. Buenos Aires.

GaBetTA, Carlos, 2003. « Crise politique totale », Manière de voir : l'Amérique latine en effervescence 69 (6-7), pp. 14-16. France, Le monde diplomatique.

Gell Alfred, 1992. "Inter-tribal Commodity Barter and Reproductive Gift-exchange in Old Melanesia », in Stephen Hugh-Jones et Caroline Humphrey (éds.), Barter, Exchange and Value, pp. 142-168. Cambridge, Cambridge University Press.

Godelier, Maurice, 1969. « La «monnaie de sel» des Baruya de Nouvelle-Guinée », L’Homme 9 (2), pp. 5-37.

Golbert, Laura et Gilbert KessLer, 2002. « Las lógicas de la violencia y la cuestión social », L'ordinaire latino américain 188 (4-6), Université de Toulouse Le Mirail, IPEALT, pp. 85-100.

GonZalez Bombal, Inès, 2002.« De la expension a la explosion : El trueque y las nuevas formas de sociabilidad en las clases medias en descenso en Argentina ", L'ordinaire latino américain 188 (4-6), Université de Toulouse Le Mirail IPEALT, pp. 81-86.

Herzfeld, Michael, 2005. Cultural Intimacy. Social Poetics in the Nation-State. New York, Routledge.

Hugh-Jones, Stephen et Caroline Humphrey, 1992. « Introduction: Barter, Exchange and Value », in Stephen Hugh-Jones \& Caroline Humphrey (éds.), Barter, Exchange and Value, pp. 1-20. Cambridge, Cambridge University Press.

Kopytoff, Igor, 1986. « The Cultural Biography of Things: Commoditization as Process », in Arjun Appadurai (dir.), The Social Live of Things: Commodities in Cultural Perspective, pp. 64-91. Cambridge, Cambridge University Press.

Mauss, Marcel, 2004. "Essai sur le don. Forme et raison de l'échange dans les sociétés archaïques », in Sociologie et anthropologie, pp. 143-279. Paris, PUF (Quadrige).

Montillet, Laurent, 2004. «Les monnaies communautaires en Argentine : le trueque. Emergence, croissance exponentielle et essoufflement de monnaies communautaires en Argentine », dans I. Guérin et J. Servet (dir.), Exclusion et liens financiers, pp. 137-158. Paris, Economica,

Ruffinelli, J. A., 1999. Introducción al trueque multirreciproco. Jarmat, Provincia Buenos Aires.

SERVET, Jean-Michel, 1998. " Démonétarisation et remonétarisation en Afrique-Occidentale et Equatoriale », dans Michel Aglietta et André Orléan (dir.), La monnaie souveraine, pp. 289-324. Paris, Odile Jacob.

Simmel, Georg, 1999. Philosophie de l'argent. Paris, PUF.

Testart, Alain, 2001. « Moyen d'échange/moyen de paiement. Des monnaies en général et plus particulièrement des primitives », dans Alain Testart (dir.), Aux origines de la monnaie, pp. 11-60. Paris, Errance.

Toffler, Alvin, 1980. La tercera ola. Barcelona, Plaza \& Jane.

ZELIZER, Viviana, 2005 [1994]. La signification sociale de l'argent. Paris, Seuil. 
